\title{
A Novel Machine Learning Approach for Detecting the Brain Abnormalities from MRI Structural Images
}

\author{
Lavneet Singh, Girija Chetty, and Dharmendra Sharma \\ Faculty of Information Sciences and Engineering \\ University of Canberra, Australia
}

\{Lavneet.singh, Girija. chetty, Dharmendra.sharma\} @ canberra.edu .au

\begin{abstract}
In this study, we present the investigations being pursued in our research laboratory on magnetic resonance images (MRI) of various states of brain by extracting the most significant features, and to classify them into normal and abnormal brain images. We propose a novel method based ondeep and extreme machine learning on wavelet transform to initially decompose the images, and then use various features selection and search algorithms to extract the most significant features of brain from the MRI images. By using a comparative study with different classifiers to detect the abnormality of brain images from publicly available neuro-imaging dataset, we found that a principled approach involving wavelet based feature extraction, followed by selection of most significant features using PCA technique, and the classification using deep and extreme machine learning based classifiers results in a significant improvement in accuracy and faster training and testing time as compared to previously reported studies.
\end{abstract}

Keywords: Deep Machine Learning, Extreme Machine Learning, MRI, PCA.

\section{Introduction}

Magnetic Resonance Images (MRI) is an advance technique used for medical imaging and clinical medicine and an effective tool to study the various states of human brain. MRI images provide the rich information of various states of brain which can be used to study, diagnose and carry out unparalleled clinical analysis of brain to find out if the brain is normal or abnormal. However, the data extracted from the images is very large and it is hard to make a conclusive diagnosis based on such raw data. In such cases, we need to use various image analysis tools to analyze the MRI images and to extract conclusive information to classify into normal or abnormalities of brain. The level of detail in MRI images is increasing rapidly with availability of 2-D and 3-D images of various organs inside the body.

Magnetic resonance imaging (MRI) is often the medical imaging method of choice when soft tissue delineation is necessary. This is especially true for any attempt to classify brain tissues [1]. The most important advantage of MR imaging is that it is non-invasive technique [2]. The use of computer technology in medical decision support is now widespread and pervasive across a wide range of medical area, such as 
cancer research, gastroenterology, heart diseases, brain tumors etc. [3, 4].Fully automatic normal and diseased human brain classification from magnetic resonance images (MRI) is of great importance for research and clinical studies. Recent work [2, 5] has shown that classification of human brain in magnetic resonance (MR) images is possible via machine learning and classification techniques such as artificial neural networks and support vector machine (SVM) [2] and unsupervised techniques such as self-organization maps (SOM) [2] and fuzzy c-means combined with appropriate feature extraction techniques [5]. Other supervised classification techniques, such as k-nearest neighbors (k-NN), which group pixels based on their similarities in each feature image $[1,6,7,8]$ can be used to classify the normal/pathological T2-wieghted MRI images.

Out of several debilitating ageing related health conditions, white matter lesions (WMLs) are commonly detected in elders and in patients with multiple brain abnormalities like Alzheimer's disease, Huntington's disease and other neurological disorders. According to previous studies, it is believed that total volume of the lesions (lesion load) and their progression relate to the aging process as well as disease process. Therefore, segmentation and quantification of white matter lesions via texture analysis is very important in understanding the impact of aging and diagnosis of various brain abnormalities. Manual segmentation of WM lesions, which is still used in clinical practices, shows the limitation to differentiate brain abnormalities using human visual abilities. Such methods can produce a high risk of misinterpretation and can also contribute to variation in correct classification. Automated texture analysis algorithms have been developed to detect brain abnormalities using image segmentation techniques and machine learning algorithms. The signal of homogeneity and heterogeneity of abnormal areas in Region of Interest (ROI) in white matter lesions of brain in T2-MRI images can be quantified by texture analysis algorithms [reference]. The ability to measure small differences in MRI images is essential and important to reduce the diagnosis errors of brain abnormalities. The supervised feature classification from T2 MRI images, however, suffers from two problems. First, because of the large variability in image appearance between different datasets, the classifiers need to be retrained from each data source to achieve good performances. Second, these types of algorithms rely on manually labeled training datasets to compute the multi-spectral intensity distribution of the white matter lesions making the classification unreliable. Inspired by new segmentation algorithms in computer vision and machine learning, we propose an efficient semi-automatic and deep learning algorithm for white matter (WM) lesion segmentation around ROI based on extreme and deep machine learning. Further, we compare this novel approach with some of the other supervised machine learning techniques reported previously.

Rest of the paper is organized as follows. Next Section gives a brief background of materials and methods used in Section 2. The details of the feature extraction, and feature selection, and other classifiers techniques used is described in same Section 2, 3 and Section 4 presents some of the experimental work carried. The paper concludes with in section 5 with some outcomes of the experimental work using proposed approach, and outlines plans for future work. 


\section{Materials and Methods}

\subsection{Coarse Image Segmentation}

Color image segmentation is useful in many applications. From the segmentation results, it is possible to identify regions of interest and objects in the scene, which is very beneficial to the subsequent image analysis or annotation. However, due to the difficult nature of the problem, there are few automatic algorithms that can work well on a large variety of data. The problem of segmentation is difficult because of image texture. If an image contains only homogeneous color regions, clustering methods in color space are sufficient to handle the problem. In reality, natural scenes are rich in color and texture. It is difficult to identify image regions containing color-texture patterns. The approach taken in this work assumes the following:

- Each region in the image contains a uniformly distributed color-texture pattern.

- The color information in each image region can be represented by a few quantized colors, which is true for most color images of natural scenes.

- The colors between two neighboring regions are distinguishable - a basic assumption of any color image segmentation algorithm.

\subsection{K-Means Clustering Based Coarse Image Segmentation}

K-Means clustering algorithm is a well-known unsupervised clustering technique to classify any given input dataset. This algorithm classifies a given dataset into discrete $\mathrm{k}$-clusters using which k-centroids are defined, one for each cluster. The next step is to take each point in the given input data set and associate it to the possible nearest centroid. This process is repeated for all the input data points, based on which next level of clustering and the respective centroids are obtained. This procedure is iterated until it converges. This algorithm minimizes the following objective function.

$$
J=\sum_{j=1}^{k} \sum_{i=1}^{k}\left\|x_{i}^{j}-c^{j}\right\|^{2}
$$

Where $\left\|x_{i}{ }^{j}-c^{j}\right\|^{2}$ is a chosen distance measure between a data point $\left(\mathrm{x}_{\mathrm{i}}\right)^{\mathrm{j}}$ and the cluster centre, $c_{j}$ is an indicator of the distance of the $\mathrm{k}$ data points from their respective cluster centers. The proposed unsupervised segmentation algorithm uses the principle of K-means clustering.

The proposed technique segments the region of interest (ROI) of an input image (input_img) by an interactive user defined shape of square or rectangle to obtain select_img. Then, the number of bins for coarse data computation (bin size), the size of overlapping kernel to partition (w-size) and the maximum number of clusters for segmentation (max_class) are fed as input data for the computation of coarse data. The coarse data identified by each kernel is aggregated to form the final_coarse_data which is further clustered using the principle of K-means clustering in order to produce the segment_img. The algorithmic description of the proposed technique is given herein under: 


\subsection{Algorithm}

1. Read a grayscale image as input_img

I* Define the area to be segmented as a runtime interactive input. The shape of the selection can either be a square or a rectangle */

2. Let select_img is the selected subimage of input_img

3. Assign:

a. binsize $=5$

/* number of bins for coarse data computation */

b. wsize $=7$

/* wsize is the size of overlapping kernel to partition the select_img */

c. max_class $=3$

/* maximum number of clusters for segmentation */

4. Repeat step 5 and 6 until the select_img is read

5. Read select_img in the order of (wsize*wsize) as window_img

6. Compute coarse_img for window_img as coarse_win_data

7. Aggregate coarse_win_data for select_img as final_coarse_data

8. Cluster final_coarse_data using K-means clustering technique using max_class in order to obtain segment_img

9. Stop

This algorithm can segment an object either fully or partially based on user's choice. If the image has a background and objects then it partitions the object from the background and displays its coarse image. If the image has no background, then the segmented image reveals the inner details of the object. This technique finds application in image processing as well as image analysis. Figure 1 shows the segmented coarse image using above mentioned algorithm.
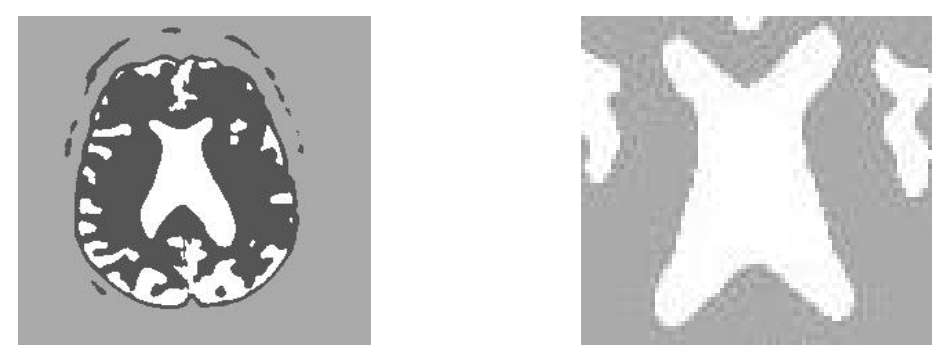

Fig. 1. (a) Coarse Segmented MRI Image based on above algorithm (b) ROI segmented image of White Lesions

\subsection{Datasets}

The input dataset consists of axial, T2-weighted, 256 X 256 pixel MR brain images (Fig. 2). These images were downloaded from the (Harvard Medical School website (http:// med.harvard.edu/AANLIB/) [9]. Only those sections of the brain in which lateral ventricles are clearly seen are considered in our study. The number of MR brain images in the input dataset is 60 of which 6 are of normal brain and 54 are of 
abnormal brain. The abnormal brain image set consists of images of brain affected by Alzheimer's and other diseases. The remarkable feature of a normal human brain is the symmetry that it exhibits in the axial and coronal images. Asymmetry in an axial MR brain image strongly indicates abnormality. A normal and an abnormal T2weighted MRI brain image are shown in Fig. 2(a), 2(b) and 2(c), respectively. Indeed, for multilayer learning models like deep and extreme machine learning algorithms needed big datasets for training, however due to lack of availability of proper datasets in MRI imaging, we used this dataset for examining the performance of proposed approaches for this paper, but acquiring other suitable datasets for future studies.

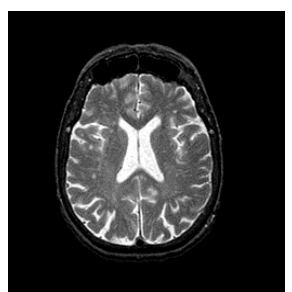

(a)

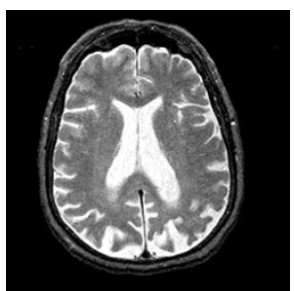

(b)

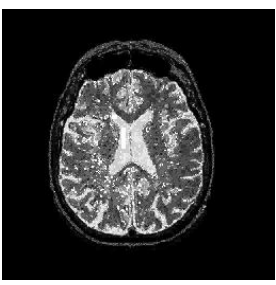

(c)

Fig. 2. (a) T2, weighted an axial MRI Brain Image; (b) T2, weighted an axial MR brain image as abnormal brain; (c) T2, weighted an axial MR brain image as normal brain after Wavelets Decomposition and denoising

\subsection{Discrete Wavelets Transform (DWT)}

The DWT is an implementation of the wavelet transform using a discrete set of the wavelet scales and translation obeying some defined rules. For practical computations, it is necessary to discretize the wavelet transform. The scale parameters are discretized on a logarithmic grid. The translation parameter $(\tau)$ is then discretized with respect to the scale parameter, i.e. sampling is done on the dyadic (as the base of the logarithm is usually chosen as two) sampling grid. The discretized scale and translation parameters are given by, $\mathrm{s}=2-\mathrm{m}$ and $\mathrm{t}=\mathrm{n} 2-\mathrm{m}$, where $\mathrm{m}, \mathrm{n} \in \mathrm{Z}$, the set of all integers. Thus, the family of wavelet functions is represented in Eq. (2) and (3),

$$
\begin{gathered}
\psi_{m, n}(t)=2^{\frac{m}{2}} \psi\left(2^{m} t-n\right) \\
W \psi(a, b)=\int_{-\infty}^{\infty} f(x) * \psi_{a, b}(t) d x
\end{gathered}
$$

In case of images, the DWT is applied to each dimension separately. This result in an image $\mathrm{Y}$ is decomposed into a first level approximation component $\mathrm{Y}_{\mathrm{a}}{ }^{1}$ and detailed components $\mathrm{Y}_{\mathrm{h}}{ }^{1} \mathrm{Y}_{\mathrm{v}}{ }^{1}$ and $\mathrm{Y}_{\mathrm{d}}{ }^{1}$ corresponding to horizontal, vertical and diagonal details. Fig. 1 depicts the process of an image being decomposed into approximate and detailed components.

The approximation component $\left(\mathrm{Y}_{\mathrm{a}}\right)$ contains low frequency components of the image while the detailed components $\left(Y_{h}, Y_{v}\right.$ and $\left.Y_{d}\right)$ contain high frequency components. Thus, 


$$
\mathrm{Y}=\mathrm{Y}_{\mathrm{a}}{ }^{1}+\left\{\mathrm{Y}_{\mathrm{h}}{ }^{1}+\mathrm{Y}_{\mathrm{v}}{ }^{1}+\mathrm{Y}_{\mathrm{d}}{ }^{1}\right\}
$$

At each decomposition level, the length of the decomposed signals is half the length of the signal in the previous stage. Hence the size of the approximation component obtained from the first level decomposition of an NXN image is $\mathrm{N} / 2 \mathrm{X} \mathrm{N} / 2$, second level is N/4 X N/4 and so on. As the level of decomposition is increased, compact but coarser approximation of the image is obtained. Thus, wavelets provide a simple hierarchical framework for interpreting the image information.

\subsection{Deep Belief Nets}

DBNs[10] are multilayer, stochastic generative models that are created by learning a stack of Restricted Boltzmann Machines (RBMs), each of which is trained by using the hidden activities of the previous RBM as its training data. Each time a new RBM is added to the stack, the new DBN has a better variation lower bound on the log probability of the data than the previous DBN, provided the new RBM is learned in the appropriate way [11].

A Restricted Boltzmann Machine (RBMs) is a complete bipartite undirected probabilistic graphical model. The nodes in the two partitions are referred as hidden and visible units. An RBM is defined as

$$
p(v, h)=\frac{e^{-E(v, h)}}{\sum_{u} \sum_{g} e^{-E(u, g)}}
$$

Where $\mathrm{v} \in \mathrm{V}$ are the visible nodes and $\mathrm{h} \in \mathrm{H}$ are the latent random variables. The energy function $\mathrm{E}(\mathrm{v}, \mathrm{h}, \mathrm{W})$ is described as

$$
E=-\sum_{i=1}^{D} \sum_{j=1}^{K} v_{i} W_{i j} h_{j}
$$

Where $\mathrm{W} \in \mathrm{R}^{\mathrm{DXK}}$ are the weights on the connections, and where we assume that the visible and hidden units both contain a node with value of 1 that acts to introduce bias. The conditional distribution for the binary visible and hidden units are defined as

$$
\begin{gathered}
p\left(v_{i}=1 / h, W\right)=\sigma\left(\sum_{j=1}^{K} W_{i j} h_{j}\right) \\
p\left(h_{j}=1 / v, W\right)=\sigma\left(\sum_{i=1}^{D} W_{i j} v_{i}\right)
\end{gathered}
$$

Where $\sigma$ is the sigmoid function. Using above equations, it easy to go back and forth between the layers of RBM. While training, it consists of some input to the RBM on the visible layer, and updating the weights and the biases such that $p(v)$ is high. In generalized way, in as set of $C$ training cases $\left\{v^{c} I c \in\{1, \ldots, C\}\right\}$, the objective is to maximize the average log probability defined as

$$
\sum_{c=1}^{C} \log \mathrm{p}\left(\mathrm{v}^{\mathrm{c}}\right)=\sum_{\mathrm{c}=1}^{\mathrm{C}} \log \frac{\sum_{\mathrm{g}} \mathrm{e}^{-\mathrm{E}\left(\mathrm{v}^{\mathrm{c}}, \mathrm{g}\right)}}{\sum_{\mathrm{u}} \sum_{\mathrm{g}} \mathrm{e}^{-\mathrm{E}(\mathrm{u}, \mathrm{g})}}
$$

The whole training process involves updating the weights with several numbers of epochs and the data is split in 20 batches which we take it randomly and the weights are update at the end of every batch. We use the binary representation of hidden units 
activation pattern for classification and visualization. The autoencoder with $\mathrm{N}_{h}$ hidden nodes is trained and fine-tuned using back-propagation to minimize squared reconstruction error, with a term encouraging low average activation of the units.

\subsection{Extreme Machine Learning}

The Extreme Learning Machine [12, 13, 14] [15] [18] is a Single hidden Layer Feed forward Neural Network (SLFN) architecture. Unlike traditional approaches such as Back Propagation (BP) algorithms which may face difficulties in manual tuning control parameters and local minima, the results obtained after ELM computation are extremely fast, have good accuracy and has a solution of a system of linear equations. For a given network architecture, ELM does not have any control parameters like stopping criteria, learning rate, learning epochs etc., and thus, the implementation of this network is very simple. Given a series of training samples $\left(\mathrm{x}_{\mathrm{i}}, \mathrm{y}_{\mathrm{i}}\right)_{\mathrm{i}=1,2 \ldots \mathrm{N}}$ and $\widehat{N}$ the number of hidden neurons where $x_{i}=\left(x_{i 1}, \ldots x_{\text {in }}\right) \in R^{n}$ and $y_{i}=\left(y_{i 1}, \ldots y_{i n}\right) \in R^{m}$, the actual outputs of the single-hidden-layer feed forward neural network (SLFN) with activation function $\mathrm{g}(\mathrm{x})$ for these $\mathrm{N}$ training data is mathematically modeled as

$$
\sum_{k=1}^{\widehat{N}} \beta_{k} g\left(\left(w_{k}, x_{i}\right)+b_{k}\right)=0_{i}, \forall=\mathrm{i}=1, \ldots ., N
$$

Where $\mathrm{w}_{\mathrm{k}}=\left(\mathrm{w}_{\mathrm{k} 1}, \ldots \ldots, \mathrm{w}_{\mathrm{kn}}\right)$ is a weight vector connecting the $\mathrm{k}^{\text {th }}$ hidden neuron, $\beta_{\mathrm{k}}=$ $\left(\beta_{\mathrm{k} 1}, \ldots \ldots \beta_{\mathrm{km}}\right)$ is the output weight vector connecting the $\mathrm{k}^{\text {th }}$ hidden node and output nodes. The weight vectors $\mathrm{w}_{\mathrm{k}}$ are randomly chosen. The term $\left(\mathrm{w}_{\mathrm{k}}, \mathrm{x}_{\mathrm{i}}\right)$ denotes the inner product of the vectors $\mathrm{w}_{\mathrm{k}}$ and $\mathrm{x}_{\mathrm{i}}$ and $\mathrm{g}$ is the activation function. The above $\mathrm{N}$ equations can be written as $\mathrm{H} \beta=\mathrm{O}$ and in practical applications $\widehat{N}$ is usually much less than the number $\mathrm{N}$ of training samples and $\mathrm{H} \beta \neq \mathrm{Y}$, where

$$
H=\left[\begin{array}{ccc}
g\left(\left(w_{1}, x_{1}\right)+b_{1}\right) & \cdots & g\left(\left(w_{\widehat{N}}, x_{1}\right)+b_{\widehat{N}}\right) \\
\vdots & \ddots & \vdots \\
g\left(\left(w_{1}, x_{1 N}\right)+b_{1}\right) & \cdots & g\left(\left(w_{\widehat{N}}, x_{N}\right)+b_{\widehat{N}}\right)
\end{array}\right]_{N X \widehat{N}}
$$

The matrix $\mathrm{H}$ is called the hidden layer output matrix. For fixed input weights $\mathrm{w}_{\mathrm{k}}=$ $\left(\mathrm{w}_{\mathrm{k} 1}, \ldots, \ldots, \mathrm{w}_{\mathrm{kn}}\right)$ and hidden layer biases $\mathrm{b}_{\mathrm{k}}$, we get the least-squares solution $\hat{\beta}$ of the linear system of equation $\mathrm{H} \beta=\mathrm{Y}$ with minimum norm of output weights $\beta$, which gives a good generalization performance. The resulting $\hat{\beta}$ is given by $\hat{\beta}=H+$ $Y$ where matrix $\mathrm{H}^{+}$is the Moore-Penrose generalized inverse of matrix $\mathrm{H}$ [14].

\subsection{Trained Classifiers and Feature Selection Evaluators}

In this study, apart from deep learning based on Restricted Boltzmann machines and extreme machine learning based on Single hidden Layer Feed forward Neural Network (SLFN) architecture as classifiers, several other classifiers are also examined in terms of accuracy and performance, including K-nearest neighbor, SVM , Naive Bayes, MultiboostAB, RotationForest, VFI, J48 and Random Forest.

To reduce the dimensionality of the large set of features of dataset, in our study, we propose the use of three optimal attribute selection algorithms: correlation based feature selection (CFS) method, which evaluates the worth of a subset of attributes by 
considering the individual predictive ability of each feature along with the degree of redundancy between them, secondly an approach based on wrappers which evaluates attribute sets by using a learning scheme. Also in this study, three search methods are also examined: the Best First, Greedy Stepwise and Scatter Search algorithms. These search algorithms are used with attribute selector's evaluators to process the greedy forward, backward and evolutionary search among attributes of significant and diverse subsets. In total, these feature selection algorithms were tested to select nearly 10 optimal and significant features out of 1024 features. The whole proposed method is implemented using Weka 3.6 platform.

\section{Experiments and Results}

\subsection{Level of Wavelet Decomposition}

We obtained wavelet coefficients of 60 brain MR images, each of whose size is $256 \mathrm{X}$ 256. Level-1 HAR wavelet decomposition of a brain MR image produces 16384 wavelet approximation coefficients; while level-2 and level-3 produce 4096 and 1024 coefficients, respectively. The preliminary experimental analysis of the wavelet coefficients through simulation in Matlab 7.10., we showed that level-2 features are the best suitable for different classifiers, whereas level-1 and level-3 features results in lower classification accuracy. We also use the DAUB-4 (Daubachies) as mother wavelets to get decomposition coefficients of MRI images at Level 2 for comparative evaluation of two wavelets decomposition methods in terms of classification accuracy.

\subsection{Attribute Selection and Classification}

In our study, three attribute or feature selection algorithms are used: correlation based feature selection (CFS) method which evaluates the worth of a subset of attributes by considering the individual predictive ability of each feature along with the degree of redundancy between them, secondly an approach based on a wrapper which evaluates attribute sets by using a learning scheme. Also in this study, three search methods are also examined: the Best First, Greedy Stepwise and Scatter Search algorithms. These search algorithms are used with attribute selector's evaluators to process the greedy forward, backward and evolutionary search among attributes of significant and diverse subsets. Table 1 shows the accuracy of classification (percentage of correctly classified samples), True Positive Rate (TP), False Positive Rate (FP) and Average Classification Accuracy (ACC) over all pair-wise combination with different feature evaluators and search algorithms with respect to multi-class classification.

Table 1 shows the performance of several learning classifiers, including K-nearest neighbor, SVM, Naive Bayes, MultiboostAB, Rotation Forest, VFI, J48 and Random Forest. Among the pair-wise classification, the lowest accuracy is observed for the classification VFI classifiers of $74.16 \%$ and the highest accuracy for the classification by Rotational forest of $97.06 \%$. Moreover, the combination of CFS feature evaluator with the of Best First search algorithm gives the highest classification accuracy.

While Table 1 shows the performance of indivual classifiers, Table 2 defines the comparative results of various combined search techniques and feature evaluators using above prescribed classifiers. Table 3 compares the proposed method against a popular 
dimensionality reduction method, known as Principal Component Analysis (PCA). PCA applies an orthogonal linear transformation that transforms data to a new coordinate system of uncorrelated variables called principal components. We have applied PCA to reduce the number of attributes or feature to 18 attributes and plotted the ROC curves using several above mentioned learning classifiers in terms of True Positive and False Positive Rate, as seen in figure 3. As can be seen in figure 3, ROC curves for all the trained learning classifiers examined in this study, the curves lie above the diagonal line describing the better classification rather than any other random classifiers. The optimal points of various trained classifiers are indicated by bold solid circles as False Positive rate (FP) and True Positive rate (TP). These optimal points in ROC curves show the maximum optimal value (FP, TP) of all trained classifiers.

Table 1. Various Classifiers comparision with respect Average Classification Accuracy(\%) and other parameters

\begin{tabular}{|c|c|c|c|c|c|c|}
\hline Classifiers & TP Rate & FP Rate & Precision & Recall & F-Measure & (ACC \%) \\
\hline KNN & 0.935 & 0.917 & 0.826 & 0.853 & 0.839 & 91.04 \\
\hline SVM & 0.912 & 0.912 & 0.831 & 0.912 & 0.87 & 91.17 \\
\hline Naive Bayes & 0.868 & 0.916 & 0.828 & 0.868 & 0.847 & 86.76 \\
\hline MultiboostAB & 0.91 & 0.91 & 0.829 & 0.91 & 0.868 & 91.04 \\
\hline Rotation Forest & 0.971 & 0.285 & 0.971 & 0.971 & 0.968 & 97.06 \\
\hline VFI & 0.742 & 0.049 & 0.93 & 0.742 & 0.796 & 74.16 \\
\hline J48 & 0.96 & 0.314 & 0.958 & 0.96 & 0.957 & 95.98 \\
\hline Random Forest & 0.97 & 0.271 & 0.97 & 0.97 & 0.968 & 97.01 \\
\hline
\end{tabular}

Table 2. Comparison of pair wise combination of various Attribute Selectors and classifiers with respect to $\mathrm{ACC}(\%)$

\begin{tabular}{|l|c|c|c|c|}
\hline \multicolumn{1}{|c|}{ Evaluator } & $\begin{array}{c}\text { Search } \\
\text { Algorithm }\end{array}$ & Classifier & N & ACC (\%) \\
\hline CFS & Best First & K-NN & 6 & 91.04 \\
\hline CFS & Greedy Stepwise & K-NN & 2 & 89.70 \\
\hline CFS & Scatter Search & K-NN & 4 & 88.23 \\
\hline Wrapper & Best First & K-NN & 5 & 89.32 \\
\hline Wrapper & Greedy Stepwise & K-NN & 4 & 87.56 \\
\hline Wrapper & Scatter Search & K-NN & 4 & 88.20 \\
\hline CFS & Best First & SVM & 6 & 91.17 \\
\hline CFS & Greedy Stepwise & SVM & 6 & 89.23 \\
\hline CFS & Scatter Search & SVM & 4 & 91.04 \\
\hline Wrapper & Best First & SVM & 2 & 90.65 \\
\hline Wrapper & Greedy Stepwise & SVM & 2 & 90.65 \\
\hline Wrapper & Scatter Search & SVM & 5 & 89.56 \\
\hline CFS & Best First & Naive Bayes & 8 & 86.76 \\
\hline CFS & Greedy Stepwise & Naive Bayes & 8 & 82.78 \\
\hline CFS & Scatter Search & Naive Bayes & 7 & 82.12 \\
\hline Wrapper & Best First & Naive Bayes & 4 & 85.44 \\
\hline Wrapper & Greedy Stepwise & Naive Bayes & 2 & 85.44 \\
\hline Wrapper & Scatter Search & Naive Bayes & 2 & 80.12 \\
\hline CFS & Best First & MultiboostAB & 5 & 91.04 \\
\hline CFS & Greedy Stepwise & MultiboostAB & 5 & 91.04 \\
\hline CFS & Scatter Search & MultiboostAB & 4 & 86.54 \\
\hline Wrapper & Best First & MultiboostAB & 5 & 89.39 \\
\hline Wrapper & Greedy Stepwise & MultiboostAB & 5 & 90.45 \\
\hline Wrapper & Scatter Search & MultiboostAB & 4 & 88.76 \\
\hline
\end{tabular}


Table 2. (Continued)

\begin{tabular}{|l|c|c|c|c|}
\hline CFS & Best First & Rotation Forest & 9 & 97.06 \\
\hline CFS & Greedy Stepwise & Rotation Forest & 9 & 96.21 \\
\hline CFS & Scatter Search & Rotation Forest & 8 & 91.66 \\
\hline Wrapper & Best First & Rotation Forest & 5 & 93.78 \\
\hline Wrapper & Greedy Stepwise & Rotation Forest & 6 & 93.78 \\
\hline Wrapper & Scatter Search & Rotation Forest & 6 & 89.54 \\
\hline CFS & Best First & VFI & 3 & 74.16 \\
\hline CFS & Greedy Stepwise & VFI & 2 & 71.01 \\
\hline CFS & Scatter Search & VFI & 4 & 71.01 \\
\hline Wrapper & Best First & VFI & 3 & 72.22 \\
\hline Wrapper & Greedy Stepwise & VFI & 2 & 72.85 \\
\hline Wrapper & Scatter Search & VFI & 4 & 72.85 \\
\hline CFS & Best First & J48 & 7 & 95.98 \\
\hline CFS & Greedy Stepwise & J48 & 7 & 95.98 \\
\hline CFS & Scatter Search & J48 & 6 & 91.41 \\
\hline Wrapper & Best First & J48 & 7 & 95.98 \\
\hline Wrapper & Greedy Stepwise & J48 & 95.98 \\
\hline Wrapper & Scatter Search & J48 & 6 & 91.41 \\
\hline CFS & Best First & Random Forest & 8 & 97.01 \\
\hline CFS & Greedy Stepwise & Random Forest & 8 & 95.47 \\
\hline CFS & Scatter Search & Random Forest & 8 & 95.47 \\
\hline Wrapper & Best First & Random Forest & 5 & 96.25 \\
\hline Wrapper & Greedy Stepwise & Random Forest & 6 & 96.25 \\
\hline Wrapper & Scatter Search & Random Forest & 5 & 90.01 \\
\hline
\end{tabular}

Table 3. Comparison using PCA and other feature attribute evaluators in terms of ACC (\%)

\begin{tabular}{|c|c|c|c|}
\hline Classifier & PCA (\%) & CFS-Best First (\%) & Wrapper-Best First (\%) \\
\hline KNN & 91.38 & 91.04 & 89.32 \\
\hline SVM & 96.24 & 91.17 & 90.65 \\
\hline Naive Bayes & 85.63 & 86.76 & 85.44 \\
\hline MultiboostAB & 94.52 & 91.04 & 89.39 \\
\hline Rotation Forest & 97.06 & 97.06 & 93.78 \\
\hline VFI & 77.12 & 74.16 & 72.22 \\
\hline J48 & 95.34 & 95.98 & 95.98 \\
\hline Random Forest & 97.34 & 97.01 & 96.25 \\
\hline
\end{tabular}

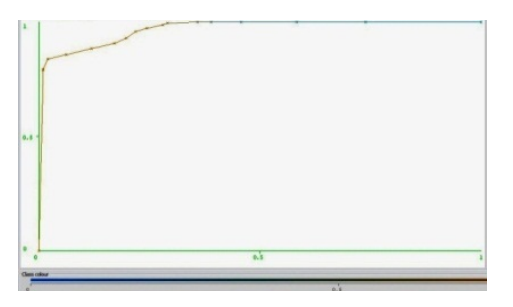

Fig. 3. Shows the ROC curve of the above mentioned trained classifiers

Table 4 describes the classification results using Extreme Machine Learning and Deep Machine Learning. In table 4, we compared the training time, testing time and classification error using extreme and deep machine Learning. As we can see in the table both learning algorithms are processed to many hidden layers and their 
evaluations is done in terms of various factors. As depicted in Table 4, it clearly shows that deep machine learning plays a major role in reducing the classification error. As Deep and extreme machine learning are designed to work on large datasets for it is difficult to compare the performance. However, they result in acceptable accuracy levels, and we are currently examining several other publicly available large MRI datasets for enhancing the performance of these two novel approaches (Deep learning and Extreme machine learning approaches).

Table 4. Classification results using Extreme Machine Learning and Deep Machine Learning

\begin{tabular}{|l|c|c|c|c|c|c|c|c|c|}
\hline & \multicolumn{3}{|c|}{ Training Time(s) } & \multicolumn{3}{c|}{ Testing Time(s) } & \multicolumn{3}{c|}{ Classification Error } \\
\hline Hidden Layers & $\mathbf{1 0}$ & $\mathbf{1 5}$ & $\mathbf{2 0}$ & $\mathbf{1 0}$ & $\mathbf{1 5}$ & $\mathbf{2 0}$ & $\mathbf{1 0}$ & $\mathbf{1 5}$ & $\mathbf{2 0}$ \\
\hline Deep Learning & 0.56 & 0.47 & 0.72 & 0.51 & 0.34 & 0.64 & 0.083 & 0.065 & 0.071 \\
\hline Extreme Learning & 0.31 & 0.31 & 0.61 & 0.41 & 0.31 & 0.56 & 0.042 & 0.042 & 0.061 \\
\hline
\end{tabular}

However, the deep learning networks do not need any particular feature reduction algorithms because of the inherent capability for feature reduction in terms of deep learning (learning through multiple layers). In case of extreme machine learning, the learning proceeds through random assignment of weights and hidden nodes (unlike gradient descendent based techniques). Due to this, there is a significant improvement in training and testing time as depicted in Table 4.

\section{Conclusions}

In this study, we have presented a principled approach for investigating brain abnormalities based on wavelet based feature extraction, PCA based feature selection and deep and extreme machine learning based classification comparative to various others classifiers. Experiments on a publicly available brain image dataset show that the proposed principled approach performs significantly better than other competing methods reported in the literature and in the experiments conducted in the study. The classification accuracy of more than $93 \%$ in case of deep machine learning and $94 \%$ in case of extreme machine learning demonstrates the utility of the proposed method. In this paper, we have applied this method only to axial T2-weighted images at a particular depth inside the brain. The same method can be employed for T1-weighted, proton density and other types of MR images. With the help of above approaches, one can develop software for a diagnostic system for the detection of brain disorders like Alzheimer's, Huntington's, Parkinson's diseases etc. Further, the proposed approach uses reduced data by incorporating feature selection algorithms in the processing loop and still provides an improved recognition and accuracy. The training and testing time for the whole study used by deep and extreme machine learning is much less as compared to SVM and other traditional classifiers reported in the literature. Further work will be pursued to classify different type of abnormalities, and to extract new features from the MRI brain images on various parameters as age, emotional states and their feedback. 


\section{References}

1. Fletcher-Heath, L.M., Hall, L.O., Goldgof, D.B., Murtagh, F.R.: Automatic segmentation of non-enhancing brain tumors in magnetic resonance images. Artificial Intelligencein Medicine 21, 43-63 (2001)

2. Chaplot, S., Patnaik, L.M., Jagannathan, N.R.: Classification of magnetic resonance brain images using wavelets as input to support vector machine and neuralnetwork. Biomedical Signal Processing and Control 1, 86-92 (2006)

3. Gorunescu, F.: Data Mining Techniques in Computer-Aided Diagnosis: NonInvasiveCancer Detection. PWASET 25, 427-430 (2007) ISSN 1307-6884

4. Kara, S., Dirgenali, F.: A system to diagnose atherosclerosis via wavelet transforms,principal component analysis and artificial neural networks. Expert Systems with Applications 32, 632-640 (2007)

5. Maitra, M., Chatterjee, A.: Hybrid multi-resolutionSlantlet transform and fuzzy c-means clustering approach for normal-pathological brain MR image segregation. Med. Eng. Phys. (2007), doi:10.1016/j.medengphy.2007.06.009

6. Abdolmaleki, P., Mihara, F., Masuda, K., DansoBuadu, L.: Neural networks analysis of astrocyticgliomas from MRI appearances. Cancer Letters 118, 69-78 (1997)

7. Rosenbaum, T., Engelbrecht, V., Krolls, W., van Dorstenc, F.A., Hoehn-Berlagec, M., Lenard, H.: MRI abnormalities in neuro-bromatosistype 1 (NF1): a study of men and mice. Brain \& Development 21, 268-273 (1999)

8. Cocosco, C., Zijdenbos, A.P., Evans, A.C.: A fully automatic and robust brainMRI tissue classification method. Medical Image Analysis 7, 513-527 (2003)

9. Database taken from, http: / / med. harvard. edu/AANLIB/

10. Hintonand, G.E., Salakhutdinov, R.R.: Reducing the dimensionality of data with neural networks. Science 313(5786), 504-507 (2006)

11. Hinton, G.E., Osindero, S.: A fast learning algorithm for deep belief nets. Neural Computation 18, 1527-1554 (2006)

12. Lin, M.-B., Huang, G.-B., Saratchandran, P., Sudararajan, N.: Fully complex extreme learning machine. Neurocomputing 68, 306-314 (2005)

13. Huang, G.-B., Zhu, Q.-Y., Siew, C.K.: Extreme Learning Machine: Theory and Applications. Neurocomputing 70, 489-501 (2006)

14. Serre, D.: Matrices: Theory and Applications. Springer Verlag, New York Inc. (2002)

15. Mishra, A., Singh, L., Chetty, G.: A Novel Image Water Marking Scheme Using Extreme Learning Machine. In: Proceedings of IEEE World Congress on Computational Intelligence (WCCI 2012). IEEE Explore, Brisbane (2012)

16. Singh, L., Chetty, G., Sharma, D.: A Hybrid Approach to Increase the Performance of Protein Folding Recognition Using Support Vector Machines. In: Perner, P. (ed.) MLDM 2012. LNCS, vol. 7376, pp. 660-668. Springer, Heidelberg (2012)

17. Singh, L., Chetty, G.: Review of Classification of Brain Abnormalities in Magnetic Resonance Images Using Pattern Recognition and Machine Learning. In: Proceedings of International Conference of Neuro Computing and Evolving Intelligence, NCEI 2012, Auckland, New-Zealand. LNCS Bioinformatics, Springer (2012)

18. Singh, L., Chetty, G.: A Novel Approach for protein Structure prediction Using Pattern Recognition and Extreme Machine Learning. In: Proceedings of International Conference of Neuro Computing and Evolving Intelligence, NCEI 2012, Auckland, New-Zealand. LNCS Bioinformatics. Springer (2012) 